\title{
Pharmacokinetic and Pharmacodynamic Properties of L-Ornithine L-Aspartate (LOLA) in Hepatic Encephalopathy
}

\author{
Gerald Kircheis ${ }^{1} \cdot$ Stefan Lüth ${ }^{1}$
}

Published online: 31 January 2019

(c) The Author(s) 2019

\begin{abstract}
L-Ornithine L-aspartate (LOLA), a stable salt of L-ornithine and L-aspartate, readily dissociates into its constituent amino acids that are readily absorbed by active transport, distributed, and metabolized. L-ornithine serves as an intermediary in the urea cycle in periportal hepatocytes in the liver and as an activator of carbamoyl phosphate synthetase, and, like L-aspartate, by transamination to glutamate via glutamine synthetase in perivenous hepatocytes as well as by skeletal muscle and brain. By way of these metabolic pathways, both amino acids participate in reactions whereby the ammonia molecule is incorporated into urea and glutamine and it is the nature, cellular, and biological location of these pathways that underpins the application of LOLA as an effective ammonia-lowering strategy widely used for the management and treatment of hepatic encephalopathy. These metabolic pathways were elucidated based upon studies in experimental animals and were confirmed by studies in patients with severe liver diseases. More recent studies suggest that LOLA may have additional direct hepatoprotective properties. Moreover, its use may result in improvements in skeletal muscle function in cirrhosis.
\end{abstract}

\section{Introduction}

L-Ornithine L-aspartate (LOLA) is a mixture of two endogenous amino acids, L-ornithine and L-aspartate. LOLA may be administered orally or parenterally. The benefits of LOLA for the lowering of blood ammonia and consequently for the treatment of hepatic encephalopathy (HE) have been known for 50 years. Within that time, numerous randomized clinical trials and an associated meta-analysis have been performed to establish the efficacy of LOLA for the treatment of HE in cirrhosis [1]. Pharmacokinetic and pharmacodynamic investigations have also been conducted. The present article will review these topics and provide an update on LOLA's mechanism of action.

Gerald Kircheis

g.kircheis@klinikum-brandenburg.de

Stefan Lüth

s.lueth@klinikum-brandenburg.de

1 Department of Gastroenterology, Hepatology and Diabetology, Brandenburg Medical School, Center of Internal Medicine II, University Hospital Brandenburg, Hochstraße 29, 14770 Brandenburg an der Havel, Germany

\section{Pharmacokinetics of L-Ornithine-L-Aspartate (LOLA)}

LOLA is a colorless crystalline powder with the molecular formula $\mathrm{C}_{9} \mathrm{H}_{19} \mathrm{~N}_{3} \mathrm{O}_{6}$ that is freely soluble in water and sparingly soluble in ethanol. LOLA, the stable salt of the naturally occurring amino acids L-ornithine and L-aspartate, is available in granular form in 5-g sachets, containing LOLA $3.0 \mathrm{~g}$ and small amounts of additional ingredients including anhydrous citric acid, lemon flavor, orange flavor, sodium saccharine, sodium cyclamate, yellow orange dye S (E 110), poly [1-vinyl-2-pyrolidone] and laevulose. LOLA is also available for injection as a $50 \%$ solution in $10.0 \mathrm{~mL}$ ampoules containing LOLA $5.0 \mathrm{~g}$ in water.

\subsection{Absorption}

LOLA dissociates into its component amino acids L-ornithine and L-aspartate, which are absorbed from the small intestine by active transport across the brush border of the intestinal epithelium. This absorption is largely dependent on the sodium ion gradient. Aspartate is carried by the dicarboxylic amino acid transport system. In the mucosal cells, conversion of glutamine, glutamate, and aspartate to alanine, citrulline, ornithine, and proline occurs. Most of the aspartate undergoes transamination with pyruvate in the mucosal 
cells of the intestinal wall, forming alanine and oxaloacetate. This reaction sharply reduces the quantity of aspartate reaching the portal blood [2]. In portal blood, most of the aspartate is found in the plasma with only a very small portion in erythrocytes. At concentrations of $1-25 \mathrm{mM}$, amino acid transporters are active just below their maximal turnover rates and net uptake via simple passive diffusion may exceed the rates via mediated pathways. In humans, normal concentrations range from 30 to $106 \mu \mathrm{mol} / \mathrm{L}$ (mean 59.8) for L-ornithine and $0-24 \mu \mathrm{mol} / \mathrm{L}$ (mean 7.5) for L-aspartate $[2,3]$.

\subsection{Single Dose Kinetics in Healthy Humans}

Intravenous (IV) administration LOLA $5 \mathrm{~g}$ in $250 \mathrm{~mL} 0.9 \%$ sodium chloride were administered intravenously to 10 healthy, fasting subjects over $30 \mathrm{~min}$ and blood levels of L-ornithine were measured at the end of infusion and hourly up to $24 \mathrm{~h}$ after infusion. A biphasic pattern was observed with a fast distribution phase $\left(\mathrm{t}_{1 / 2} 15-25 \mathrm{~min}\right)$ and a slower terminal elimination phase $\left(t_{1 / 2} 120-150 \mathrm{~min}\right)$. Peak levels of L-ornithine $30 \mathrm{~min}$ after the beginning of the infusion were nearly 10 times basal levels that fell to normal within $7 \mathrm{~h}$. The median area under the curve (AUC) for L-ornithine was $1.390 \mathrm{mmol} / \mathrm{h} / \mathrm{L}$ [4].

Oral administration LOLA $5 \mathrm{~g}$ in $250 \mathrm{~mL}$ water were consumed by 10 healthy, fasting subjects and blood levels determined at the same intervals as the intravenous (IV) infusions (above). L-ornithine peaked within 30-60 min to levels five times greater than basal concentrations and returned to baseline within $7 \mathrm{~h}$. The median AUC for L-ornithine was $1.143 \mathrm{mmol} / \mathrm{h} / \mathrm{L}$. Bioavailability was $82.2 \pm 28 \%$ after IV or oral administration [4].

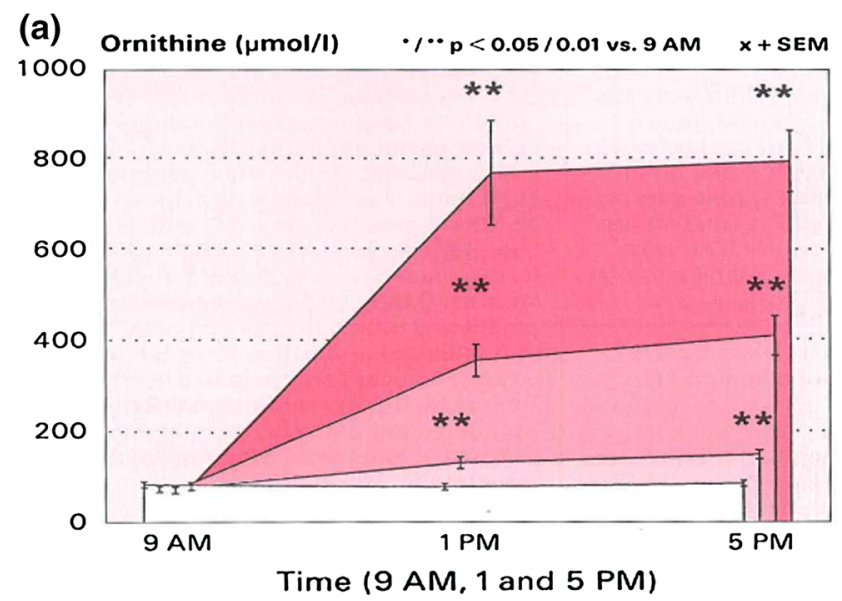

Fig. 1 Pharmacokinetics of L-ornithine L-aspartate (LOLA) in patients with cirrhosis, hyperammonemia and hepatic encephalopathy. a Plasma levels of L-ornithine $4 \mathrm{~h}$ and $8 \mathrm{~h}$ post-infusion of 5-20 g LOLA versus placebo. b Plasma levels of L-aspartate $4 \mathrm{~h}$

\subsection{Intravenous Administration to Cirrhotic Patients}

In a double-blind, randomized, placebo-controlled, crossover clinical trial, 10 cirrhotic patients with HE and hyperammonemia were studied on four separate days with an interval of 2-5 days between single infusions of a range of doses of LOLA. After an overnight fast, the patients consumed a liquid protein formula of $0.25 \mathrm{~g} / \mathrm{kg}$ at 9 a.m. and $0.50 \mathrm{~g} / \mathrm{kg}$ at $1 \mathrm{p} . \mathrm{m}$. Blood was drawn for analysis of ammonia and amino acids immediately before and every $2 \mathrm{~h}$ after infusion. Doses of LOLA were $0 \mathrm{~g}$ (vehicle only), $5 \mathrm{~g}, 20 \mathrm{~g}$, and $40 \mathrm{~g}$ starting at $9 \mathrm{a} . \mathrm{m}$. and continuing for $8 \mathrm{~h}$. The dose of LOLA was selected randomly [5].

Following continuous infusion of placebo $(0.9 \% \mathrm{NaCl})$, concentrations of L-ornithine (Fig. 1a) and L-aspartate (Fig. 1b) remained stable for the entire 8-h period. Following LOLA $5 \mathrm{~g}(0.625 \mathrm{~g} / \mathrm{h})$, the concentration of L-ornithine increased to $132 \pm 13 \mu \mathrm{mol} / \mathrm{L}$ (4-h infusion) and $148 \pm 10 \mu \mathrm{mol} / \mathrm{L}$ (8-h infusion) while that of L-aspartate increased to a near steady state level $(11 \pm 2 \mu \mathrm{mol} / \mathrm{L}$ after 4 to 8 -h infusion). Continuous administration of $2.5 \mathrm{~g} / \mathrm{h} \mathrm{LOLA}$ $(20 \mathrm{~g} / 8 \mathrm{~h})$ increased the level of L-ornithine and L-aspartate 4 -fold whereas $5 \mathrm{~g} / \mathrm{h} \mathrm{LOLA}(40 \mathrm{~g} / 8 \mathrm{~h})$ led to a 10 -fold increase of L-ornithine and a 20 -fold increase of L-aspartate above basal placebo levels (Fig. 1a, b [5]).

\subsection{Distribution and Metabolism}

Orally administered LOLA promptly splits into L-ornithine and L-aspartate in the upper gut [6]. Subsequently, via transamination reactions, the amino nitrogen of the various

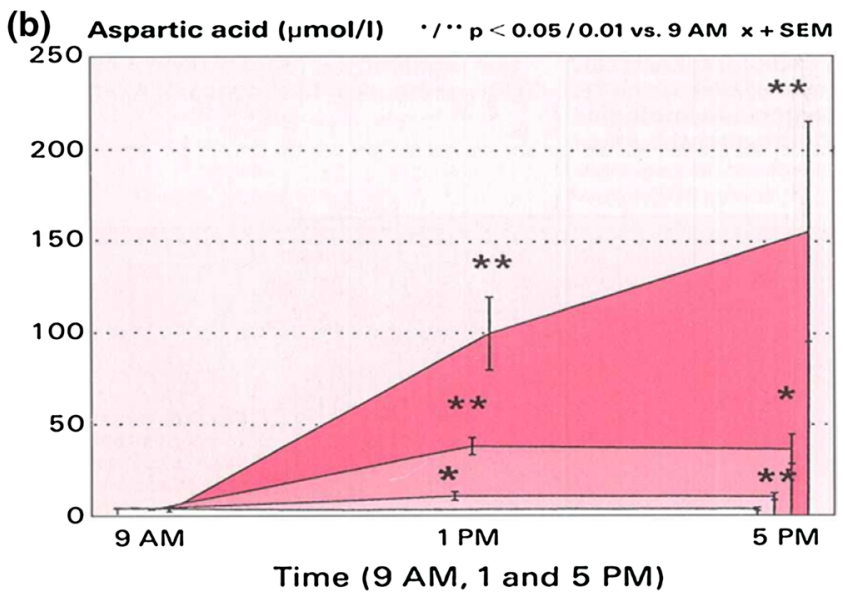

and $8 \mathrm{~h}$ post-infusion of 5-40 g LOLA versus placebo. Intensity of shading indicates increasing doses of LOLA. Error bars indicate mean \pm SEM; values significantly different from baseline ( 9 a.m. values) indicated by $* p<0.05, * * p<0.01$ by ANOVA 
amino acids may be incorporated into alanine, aspartate, and glutamate. Dependent on the patient's status, it may then be reused for the biosynthesis of protein or converted to urea for purposes of excretion. Alanine is utilized in protein synthesis and other reactions and oxaloacetate is oxidized either via the tricarboxylic acid cycle or via aspartate [7]. Some residual aspartate may combine with citrulline to form arginine-succinate that is subsequently cleaved to fumarate and arginine or combine with carbamyl-phosphate to initiate pyrimidine synthesis. The remaining aspartate, and newly formed malate, $\alpha$-ketoglutarate, and oxaloacetate, are taken up by the perivenous scavenger cells where they serve as the carbon source for glutamine synthesis.

After reaching the portal blood, L-ornithine is directly taken up by the periportal hepatocytes of the liver and metabolized by the mitochondria [8]. The ornithine serves as an intermediary in the urea cycle and is an activator of carbamyl-phosphate synthetase, the rate-limiting enzyme of urea synthesis [9]. Some ornithine is decarboxylated and incorporated into polyamines; some undergoes transamination to form glutamate semialdehyde and glutamate, a reaction that generates NADH. L-Ornithine is continuously regenerated in the urea cycle and the amino group donor, L-aspartate, can be regenerated in a few enzymatic steps. Thus, during urea biosynthesis, the hepatocyte loses two ammonia molecules as well as bicarbonate and energy. L-ornithine is converted into glutamate-y-semialdehyde that is dehydrated to form the dicarboxylic acid. Thus, through reversal of the routes of metabolism and different enzymes, L-ornithine, on the one hand, can be synthesized, and, on the other hand, can be decarboxylated to form the diamine putrescine.

\subsection{Excretion}

LOLA is not excreted as such but urea, its major product, is excreted in the urine. Flux through the urea cycle is controlled by extracellular $\mathrm{pH}$, primarily by bicarbonate and $\mathrm{CO}_{2}$. A decrease of urea synthesis in acidosis is followed by bicarbonate sparing and the excretion of ammonium ions into urine (renal ammonia-genesis) [7]. Glutamine serves in the transport of ammonia from liver to kidney. In acidosis, flux through the urea cycle and hepatic glutaminase is decreased, whereas flux through hepatic glutamine synthetase and renal glutaminase is increased $[9,10]$.

\subsection{Toxicity}

Acute oral administration of LOLA to rats and mice results in no measurable toxicity even after the maximal applied dose of $\sim 5.6 \mathrm{~g} / \mathrm{kg}$. After IV administration, the lethal dose, $50 \%\left(\mathrm{LD}_{50}\right)$ ranged from $3.1 \mathrm{~g} / \mathrm{kg}$ in mice to $4.7 \mathrm{~g} /$ $\mathrm{kg}$ in rats. There were no pathological abnormalities at autopsy in either group [11]. Chronic toxicity after repeated administration of LOLA was tested sub-chronically and chronically in animal models. In a pilot study of sub-chronic toxicity in rats, LOLA was mixed in the diet in concentrations of $0.5-1 \%$. Administered doses of LOLA were as high as $1 \mathrm{~g} / \mathrm{kg}$. No treatment-related abnormalities to LOLA were observed [11]. The toxicity of IV LOLA $(1-4 \mathrm{~g} / \mathrm{kg})$ after administration for $6 \mathrm{~h} /$ day for 4 weeks was investigated in Sprague-Dawley (SD) rats. All doses caused local irritation at the site of injection but there were no signs of systemic toxicity.

In Beagle dogs, doses of 0.6-3.75 g/kg IV LOLA were administered ( $6 \mathrm{~h} /$ day for 4 weeks). No toxicity was observed at the $0.6 \mathrm{~g} / \mathrm{kg}$ dose but at $1.5 \mathrm{~g} / \mathrm{kg}$, increased salivation and slightly decreased body weight occurred. At $3.75 \mathrm{~g} / \mathrm{kg}$, animals experienced repeated vomiting. No other differences between placebo- and LOLA-treated animals were seen in terms of clinical findings, hematology, blood chemistry, urinalysis or histopathology [12].

\subsubsection{Mutagenesis}

LOLA was tested for mutagenic potential in four different ways, namely the AMES test, the Micronucleus test, the Gen-mutation test as well as the Chromosome-aberration test. In none of these tests was any mutagenic potential detected [13].

\subsubsection{Carcinogenesis}

L-Ornithine and L-aspartate are naturally occurring amino acids and are common ingredients of human diets. They are integral components of human amino acid metabolism. These facts, together with the proven non-mutagenic potential of LOLA [14], exclude the possibility of any significant carcinogenic potential.

\subsubsection{Reproductive Performance}

Male and female rats were administered LOLA once daily by gavage at doses of $0.5-3 \mathrm{~g} / \mathrm{kg}$ for 2 weeks prior to mating, during mating and, for females, during pregnancy and lactation. There were no apparent effects on growth, development or behavior of the offspring when observed until the age of 35 days [15].

\section{Pharmacodynamics of LOLA}

Investigations of the effects of LOLA on hyperammonemia induced in experimental animals have been reported over a period of several decades. Investigations in the 1980s and 
1990s focused on the details of the working mechanism of LOLA.

\subsection{Ammonia-Lowering Effect and Urea Synthesis}

Investigations showed that L-ornithine in doses of $4 \mathrm{mmol} / \mathrm{kg}$ body weight protected rats given $\mathrm{LD}_{99.9}$ doses of ammonium acetate. They demonstrated that the effects of intraperitoneally injected L-ornithine were mediated by the acceleration of urea synthesis in the liver. In rats poisoned with carbon tetrachloride, LOLA was more effective than L-ornithine alone [16]. In other studies, L-ornithine and L-aspartate protected against the hepatotoxic effects of carbon tetrachloride, and L-ornithine and L-aspartate in combination were more effective than either agent alone. A reduction of blood ammonia levels and increase in serum urea levels in rats given $\mathrm{LD}_{50}$ doses of ammonium acetate were also reported [17]. LOLA (3 mL/kg, intramuscularly [IM]) given to rabbits administered $50 \%$ carbon tetrachloride or LOLA $(5 \mathrm{~mL} /$ $\mathrm{kg}$ IV) given to rats treated with $5.6 \%$ ammonium sulfate resulted in normalization of impaired ammonia metabolism. The authors concluded that LOLA administration is more effective than L-aspartate alone [18]. Others reported that pretreatment with LOLA effectively attenuated the toxicity and mortality in mice or rabbits given very high doses of ammonium salts. Pretreatment with LOLA was more effective than L-ornithine or L-aspartate alone [11].

\subsection{Mode of Ammonia-Lowering Actions of LOLA}

LOLA provides critical substrates for both ureagenesis and glutamine synthesis. Urea synthesis is an irreversible, liver-specific process, located primarily in the periportal hepatocytes [10]. L-Ornithine stimulates flux through the carbamylphosphate synthetase pathway. L-Ornithine, which is also an activator of ornithine carbamyltransferase and a substrate of urea synthesis, is almost entirely involved in the activation of the urea cycle leading to the detoxification of ammonia, particularly in conditions of impaired urea cycle enzyme activities as in cirrhotic patients [10].

\subsubsection{Pharmacodynamic Effects of LOLA on Ammonia Metabolism}

The effects of LOLA on hyperammonemia and urea metabolism in cirrhotic rats were extensively investigated in additional experimental animal studies [19]. Cirrhosis was induced by carbon tetrachloride $\left(\mathrm{CCl}_{4}\right)$. In cirrhotic animals, the activities of carbamoyl phosphate synthetase and arginase were lowered, indicating reduced functioning of the urea cycle. Administration of LOLA significantly increased the activity of these enzymes by $30 \%$ and $40 \%$, respectively. At the same time, there was a significant increase in urea production. The decrease in ammonia concentration was also significant. It was concluded from these results that treatment of cirrhotic rats with LOLA increases urea synthesis (Fig. 2) and, thus, the capacity of residual periportal hepatocytes to detoxify ammonia [19].

\subsubsection{Ammonia-Lowering Effect of LOLA in Cirrhosis-Related Hepatic Encephalopathy (HE)}

Studies of the effects of an infusion of LOLA $(2.8 \mathrm{mmol} /$ $\mathrm{kg} / \mathrm{h}$ in ammonium acetate-intoxicated portacaval shunted rats resulted in a 1.8-fold increase of urea production leading to reduction of blood ammonia concentration and a $25 \%$ reduction of brain ammonia concentrations [20]. Plasma concentrations of glutamate, glutamine, arginine, alanine, ornithine, and aspartate were significantly increased in LOLA-treated rats compared with controls. Extracellular brain glutamine concentrations were increased 9-fold in LOLA-treated rats in contrast to 1.2 -fold increases in controls [20].

A subsequent study from in 1994 in the same animal model carried out by in vivo proton magnetic resonance

\section{NORMAL}

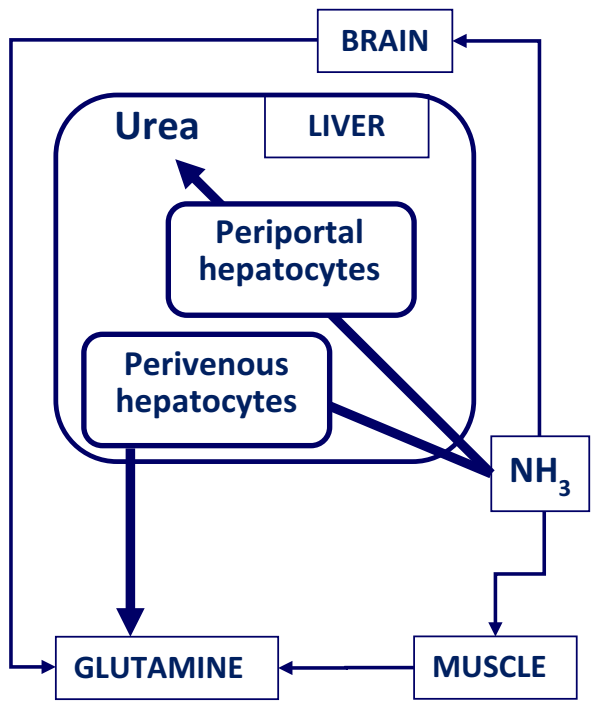

Fig. 2 Inter-organ trafficking of ammonia under normal physiological conditions. Ammonia produced principally from protein digestion in the gastrointestinal tract or in part from glutamine via glutaminase in the kidney is removed in the liver as urea by periportal hepatocytes or as glutamine by perivenous hepatocytes. Ammonia is transported to the brain and to skeletal muscle where the sole mechanism for ammonia removal is via glutamine synthesis. In severe liver disease, hepatic ammonia removal capacity is decreased and skeletal muscle takes over as the major ammonia-removing organ. This pathway may be compromised by muscle wasting (sarcopenia) in cirrhosis 
spectroscopy (1H-MRS) revealed a reduction in brain ammonia following administration of LOLA, as well as attenuation of the increase in brain glutamine compared with controls [21].

LOLA infusions prevented ammonium acetate-induced coma in all portacaval-shunted animals, none of which showed deterioration of neurological status. Protective effects of LOLA were accompanied by smaller increases in blood ammonia and increased urea concentration compared with controls. Concentrations of glutamine in the brain were significantly higher than in the control animals, indicative of increased brain removal of ammonia by LOLA [22].

\subsubsection{Effects of LOLA on HE in Acute Liver Failure}

Brain edema and brain herniation remain major causes of mortality in acute liver failure (ALF). LOLA was used to evaluate the therapeutic efficacy in groups of rats with ALF caused by hepatic devascularization. Intravenous infusions of LOLA $(0.33 \mathrm{~g} / \mathrm{kg} / \mathrm{h})$ resulted in normalization of plasma ammonia concentrations and in a significant delay in onset of severe HE. More importantly, brain edema was significantly reduced in LOLA-treated ALF rats. These protective effects of LOLA were accompanied by increased plasma concentrations of glutamate, GABA, taurine, and alanine, as well as branched-chain amino acids (leucine, isoleucine, and valine). Increased availability of glutamate following LOLA treatment provides the substrate for glutamine synthetase, the major ammonia-removal enzyme. Plasma glutamine concentrations were increased 2-fold in LOLA-treated ALF rats consistent with increased muscle glutamine synthesis, and direct measurement of glutamine synthetase activities in muscle revealed a 2-fold increase following LOLA treatment. These findings demonstrate a significant ammonialowering effect of LOLA together with a protective effect on the development of the cerebral complications (HE and brain edema) in ALF [23].

\subsubsection{Role of Skeletal Muscle in the Ammonia-Lowering Effects of LOLA in HE}

In an unselected series of patients who had emergency portacaval shunt surgery, severe muscle wasting (sarcopenia) was associated with a worse prognosis for survival compared with any other currently available indicator [24, 25].

In a ${ }^{13} \mathrm{NH}_{3}$ whole body tracer study in a series of patients with cirrhosis, some of whom had HE, the rates of uptake of ammonia into skeletal muscle as well as the release of glutamine from the muscle were found to be significantly increased [25].

Subsequent studies in rats with end-to-side portacaval anastomoses (an animal model of type B HE) revealed that activities of the enzyme glutamine synthetase were increased
[26] resulting from a post-translational modification of the glutamine synthetase gene [27]. Taken together, these findings support the notion that, in cirrhosis and/or portal-systemic shunting, skeletal muscle takes over from the failing liver as the primary organ responsible for the removal of excess ammonia.

The effects of LOLA on muscle protein synthesis and breakdown, as well as leucine turnover and incorporation into muscle, have been investigated using stable isotope techniques in cirrhotic patients with hyperammonemia and sarcopenia [28, 29]. Forearm-blood exchange was studied and muscle biopsies from the quadriceps or anterior tibialis muscle were taken under local anesthesia at specific time points [29]. A single infusion of LOLA $5 \mathrm{~g} / \mathrm{h}$ was ineffective but continued LOLA infusions over 7 days restored the responsiveness of muscle metabolism to the protein meal and protein catabolism was inhibited by repeated LOLA infusions.

These findings have been taken one step further recently with the publication of the findings of a study in which lowering of blood ammonia by a combination of LOLA and the antibiotic rifaximin resulted in restoring skeletal muscle proteostasis in portacaval-shunted rats, leading to the proposal that ammonia-lowering strategies such as the use of LOLA have the potential to reverse sarcopenia in cirrhosis [30].

\subsubsection{Hepatoprotective Effects of LOLA in Cirrhosis}

Reports of attenuation of increases in liver enzymes, bilirubin, and prothrombin times as well as improvements in Child-Pugh and MELD scores continue to appear in the literature based upon studies of the effects of LOLA in patients with cirrhosis [31]. In most cases, improvements in markers of hepatic function were accompanied by reductions of hyperammonemia and improvements in HE grades.

Two major mechanisms have been proposed to explain the hepatoprotective actions of LOLA in chronic liver disease. These mechanisms include the antioxidant properties of glutathione (GSH) synthesized from L-ornithine via transamination to glutamate [32] as well as increased synthesis of nitric oxide (NO) from the increased production of L-arginine from L-ornithine via elements of the urea cycle. Previous studies in patients with cirrhosis [5] as well as in an experimental model of chronic liver failure $[22,23]$ confirm that administration of LOLA does indeed result in the accumulation of L-glutamate and L-arginine. Since L-arginine is the obligate substrate for NO synthase (NOS), increases in its availability would be expected to result in increased NOS with consequent increases in hepatic microperfusion [33]. Clearly, improvements in hepatic function would explain the reported increases in ammonia-removal capacity, thus providing an additional, albeit indirect, mechanism of action of LOLA. 


\section{Conclusion}

Systematic pharmacokinetic and pharmacodynamic studies in experimental models of liver failure and in patients have resulted in a clear understanding of the mechanisms that underpin the effective ammonia-lowering actions of LOLA. Transformations of the constituent amino acids of LOLA either via the hepatic urea cycle into urea per se or into glutamine via glutamine synthetase expressed by perivenous hepatocytes, brain, and skeletal muscle afford multiple pathways whereby ammonia is readily removed. The efficacy of LOLA for ammonia reduction and concomitant improvements in HE has been substantiated in randomized clinical trials and a meta-analysis. Moreover, recent studies provide evidence to suggest that LOLA may also have hepatoprotective properties and may assist in the prevention of sarcopenia in patients with cirrhosis.

Funding This article is published in a special edition journal supplement wholly funded by Merz Pharmaceuticals GmbH, Frankfurt, Germany.

\section{Compliance with Ethical Standards}

Conflict of Interest Dr Gerald Kircheis has received a research grant from Merz Pharmaceuticals and received speaker honoraria from Merz Pharmaceuticals and Norgine. Prof. Stefan Lüth has received speaker honoraria or research grants from Alexion, Abbvie GmbH Co. KG, BMS, Gilead, Intercept, Lilly, Merz Pharmaceuticals, MSD, Novartis, Roche, and Shire.

Open Access This article is distributed under the terms of the Creative Commons Attribution-NonCommercial 4.0 International License (http://creativecommons.org/licenses/by-nc/4.0/), which permits any noncommercial use, distribution, and reproduction in any medium, provided you give appropriate credit to the original author(s) and the source, provide a link to the Creative Commons license, and indicate if changes were made.

\section{References}

1. Butterworth RF, Kircheis G, Hilger N, McPhail MJW. Efficacy of L-ornithine L-aspartate for the treatment of hepatic encephalopathy and hyperammonemia in cirrhosis: systematic review and meta-analysis of randomized controlled trials. J Clin Exp Hepatol. 2018;8:301-13.

2. Schultz SG, Curran PF. Coupled transport of sodium and organic solutes. Physiol Rev. 1970;50:637-718.

3. Stevens BR. Amino acid transport in intestine. In: Dilberg MS, Häussinger D, editors. Mammalian amino acid transport. New York: Plenum Press; 1992. pp. 149-163.

4. Merz and Co. Scientific report MRZ 90004-9002. Deternination of the absolute bioavailability of Hepa Merz in 10 subjects. MERZ Internal Report; 1991. pp. 1-17.

5. Staedt U, Leweling H, Gladisch R, Kortsik C, Hagmüller E, Holm E. Effects of ornithine aspartate on plasma ammonia and plasma amino acids in patients with cirrhosis. A double-blind, randomized study using a four-fold crossover design. J Hepatol. 1993;19:424-30.

6. Munro HN, Crim MC. Modern nutrition in health and disease. In: Shils ME, Young VR, editors. 7th edn., chap 1; 1988. pp. 1-37.

7. Saheki T, Hosoya M, Fujinami S, Katsunuma T. Regulation of urea synthesis: changes in the concentration of ornithine in the liver corresponding to changes in urea synthesis. Adv Exp Med Biol. 1982;153:255-63.

8. Hommes FA, Kitchings L, Eller AG. The uptake of ornithine and lysine by rat liver mitochondria. Biochem Med. 1983;30:313-21.

9. Knepper MA, Packer R, Good DW. Ammonium transport in the kidney. Physiol Rev. 1989;69:179-249.

10. Häussinger D, Steeb R, Kaiser S, Wettstein M, Stoll B, Gerok W. Nitrogen metabolism in normal and cirrhotic liver. Adv Exp Med Biol. 1990;272:47-64.

11. Shioya A, Kuraishi K, Kakimoto M, Tamama Y. Pharmacological study on L-ornithine-L-aspartate. Jpn J Pharmacol. 1964;14:201-14.

12. LPT, Hamburg. Project 6699/91: Twenty six week chronic toxicity study of ornithine-aspartate by oral administration to beagle dogs. MERZ Internal Report; 1991.

13. Sargentini NJ, Smith KC. Mutagenesis by normal metabolites in Escherichia coli: phenylalanine mutagenesis is dependent on error-prone DNA repair. Mutat Res. 1986;161:113-8.

14. Chany C, Cerutti Z. Aspartate-assisted immune stimulation: its importance in antitumor and antiviral protection. Int $\mathrm{J}$ Cancer. 1986;38:259-64.

15. Toxicol GB. MEZ/6/R: Oral (gavage) rat general reproductive performance dose-ranging study (Internal Document).

16. Greenstein JP, Winitz M, Gullino P, Otey MC, Winitz M. Studies on the metabolism of amino acids and related compounds in vivo. III. Prevention of ammonia toxicity by arginine and related compounds. Arch Biochem. 1956;64:342-54.

17. Salvatore F, Scoppa P, Cozzolino D. Protective effect of ornithine and aspartic acid in chronic carbon tetrachloride intoxication. Clin Chim Acta. 1959;4:728-32.

18. Yoshida T, Koizumi T, Kojima Y. Influence of L-ornithineL-aspartate on ammonia metabolism in rabbits with carbon tetrachloride hepatic disorder. Tokyo: Kyowa Hakko Kogyo Co., Ltd.; 1963. p. 1-3 (Internal Document).

19. Gebhardt R, Beckers G, Gaunitz F, Haupt W, Jonitza D, Klein $\mathrm{S}$, et al. Treatment of cirrhotic rats with L-ornithine-L-aspartate enhances urea synthesis and lowers serum ammonia levels. J Pharmacol Exp Ther. 1997;283:1-6.

20. Vogels BA, Karlsen OT, Mass MA, Boveé WM, Chamuleau RA. L-Ornithine vs. L-ornithine-L-aspartate as a treatment for hyperammonemia-induced encephalopathy in rats. J Hepatol. 1997;26:174-82.

21. Slotboom J, Vogels BAPM, De Haan JG, et al. Proton resonance spectroscopy study of the effects of L-ornithine L-aspartate on the development of encephalopathy, using localization pulses with reduced specific absorption rate. J Mag Res. 1994;105:147-56.

22. Rose C, Michalak A, Pannunzio P, Therrien G, Quack G, Kircheis $\mathrm{G}$, et al. L-ornithine-L-aspartate in experimental portal-systemic encephalopathy: therapeutic efficacy and mechanism of action. Metab Brain Dis. 1998;13:147-57.

23. Rose C, Michalak A, Rao KVR, Quack G, Kircheis G, Butterworth RF. L-Ornithine-L-Aspartate lowers plasma and cerebrospinal fluid ammonia and prevents brain edema in rats with acute liver failure. Hepatology. 1999;30:636-40.

24. Morrison WL, Bouchier IAD, Gibson JNA, et al. Skeletal muscle and whole-body turnover in cirrhosis. Clin Sci. 1990;78:613-9.

25. Reynolds N, Downie S, Smith K, Kircheis G, Rennie MJ. Treatment with L-ornithine-L-aspartate infusion restores muscle protein 
synthesis responsiveness to feeding in patients with cirrhosis. $\mathbf{J}$ Hepatol. 1999;30:65 (abstract).

26. Orloff MJ, Charters AC, Chandler JC, Condon JK, Grambort DE, Modafferi TR, et al. Portacaval shunt as emergency procedure in unselected patients with alcoholic cirrhosis. Surg Gynecol Obstet. 1975;141:59-68.

27. Lockwood AH, McDonald JM, Reiman RE, Gelbard AS, Laughlin JS, Duffy TE, et al. The dynamics of ammonia metabolism in man: effects of liver disease and hyperammonemia. J Clin Invest. 1979;63:449-60.

28. Girard G, Butterworth RF. Effect of portacaval anastomosis on glutamine synthetase activities in liver, brain and skeletal muscle. Digest Dis Sci. 1992;37:1121-6.

29. Desjardins P, Rama Rao KV, Michalak A, Rose C, Butterworth RF. Effect of portacaval anastomosis on glutamine synthetase protein and gene expression in brain, liver and skeletal muscle. Metab Brain Dis. 1999;14:273-80.
30. Kumar A, Davuluri G, Silva RNE, Engelen MPKJ, Ten Have GAM, Prayson R, et al. Ammonia lowering reverses sarcopenia of cirrhosis by restoring skeletal muscle proteostasis. Hepatology. 2017;65:2045-58.

31. Butterworth RF, Gruengreiff K. L-ornithine L-aspartate (LOLA) for the treatment of hepatic encephalopathy in cirrhosis: evidence for novel hepatoprotective mechanisms. J Liver Clin Res. 2018;5:1044.

32. Najmi AK, Pillai KK, Pal SN, Akhtar M, Aqil M, Sharma M. Effect of L-ornithine L-aspartate against thioacetamide-induced hepatic damage in rats. Ind J Pharmacol. 2010;42:384-7.

33. Ijaz S, Yang W, Winslet MC, Seifalian AM. The role of nitric oxide in the modulation and tissue oxygenation in an experimental model of hepatic steatosis. Microvasc Res. 2005;70:129-36. 